Revue interdisciplinaire sur la Grèce archaïque

\title{
Encolpio e Ascilto, all'ombra di Epimeteo. Nota a Petronio, Satyricon, 7, 4
}

Encolpe et Ascylte, à l'ombre d'Epiméthée. Une note à Pétrone, Satyricon, 7, 4

Encolpius and Ascyltos, in the Shadow of Epimetheus. A Note to Petronius'

Satyricon, 7, 4

\section{Gian Franco Gianotti}

\section{(2) OpenEdition}

\section{Journals}

Edizione digitale

URL: https://journals.openedition.org/gaia/2094

DOI: $10.4000 /$ gaia.2094

ISSN: 2275-4776

Editore

UGA Éditions/Université Grenoble Alpes

Edizione cartacea

ISBN: 978-2-37747-292-5

ISSN: $1287-3349$

Notizia bibliografica digitale

Gian Franco Gianotti, «Encolpio e Ascilto, all'ombra di Epimeteo. Nota a Petronio, Satyricon, 7, 4», Gaia

[Online], 24 | 2021, online dal 31 mai 2021, consultato il 09 décembre 2021. URL: http://

journals.openedition.org/gaia/2094 ; DOI: https://doi.org/10.4000/gaia.2094

Questo documento è stato generato automaticamente il 9 décembre 2021.

Gaia. Revue interdisciplinaire sur la Grèce archaïque 


\section{Encolpio e Ascilto, all'ombra di Epimeteo. Nota a Petronio, Satyricon, 7, 4}

Encolpe et Ascylte, à l'ombre d'Epiméthée. Une note à Pétrone, Satyricon, 7, 4 Encolpius and Ascyltos, in the Shadow of Epimetheus. A Note to Petronius' Satyricon, 7, 4

\section{Gian Franco Gianotti}

\section{NOTE DELL'AUTORE}

Mi è caro dedicare queste pagine alla memoria di Ezio Pellizer, nel ricordo dei seminari triestini sulla ricezione romana di temi e motivi ellenici, svolti insieme a Ezio e in compagnia di altri amici e colleghi: Mario Martina (1948-1998), Gennaro Tedeschi, Innocente Toppani (1943-2021), Nevio Zorzetti. Un grazie sentito ai revisori anonimi, a Lucio Bertelli, Manuela Callipo e Sonia Francisetti Brolin per i suggerimenti e l'aiuto di cui sono stati prodighi nei miei confronti.

1 Nel frammento per noi iniziale del testo petroniano la discussione de causis corruptae eloquentiae si apre in medias res e la persona loquens, da identificare con Encolpio, mette sotto accusa gli sproloqui verbosi dei declamatori. I risultati di tali eccessi - si sostiene - non aprono ai futuri oratori la via che porta all'eloquenza; sortiscono invece effetto contrario, perché il turgore enfatico delle argomentazioni e le rumorose assurdità del vaniloquio dei cattivi maestri fanno sì che i giovani, «una volta approdati al foro, pensino di esser stati sbalestrati in un altro mondo» ${ }^{1}$. Per quanto è possibile ricavare dall'incompleta conservazione del Satyricon, la formula sembra avere valore programmatico e condannare i due protagonisti, scholastici veri o fittizi, alle alterne vicende degli errori di valutazione e della comprensione tardiva. 
2 Il primo a farne le spese è Encolpio: accortosi che Ascilto si è allontanato, abbandona il retore Agamennone e l'ingens scholasticorum turba appena sopraggiunta, per inseguire il sodale, pericoloso rivale d'amore; non ritrova però la via e finisce in un virtuale labirinto cittadino, perché ritorna sempre sui propri passi e deve constatare di non sapere più («nec [...] sciebam») l'ubicazione della locanda in cui alloggiava con l'amico e il giovane amasio ${ }^{2}$. Il tentativo di porre rimedio alla temporanea ignoranza è davvero singolare e curioso: il giovane si avvicina a una vecchierella, intenta a vendere ortaggi, e chiede con garbo sciocco e un po' surreale che non manca di divertire la donna: «Ti prego, mammina, sai forse (numquid scis) dove abito?» ${ }^{3}$. La risposta non è meno surreale: «Come potrei non saperlo? (quidni sciam?)». Dunque, rispetto all'ignoranza di Encolpio, la vecchietta appare depositaria, per esperienza dovuta all'età e per ammissione stessa di chi ha formulato la stolida richiesta, di una scientia del tutto specifica, in quanto afferma di sapere quale sia l'abitazione appropriata per giovanetti solitari e inquieti; guida infatti Encolpio verso un luogo piuttosto appartato, scosta una tenda di scarso valore e sentenzia con sicurezza: «hic debes habitare». Il giovane nega lì per lì di riconoscere la dimora indicata («cum ego negarem me agnoscere domum»), ma dalla presenza di scritte non ambigue ${ }^{4}$ e meretrici ignude, tra cui s'aggirano personaggi con fare guardingo, comprende finalmente d'esser stato condotto in un postribolo: «tarde, immo iam sero intellexi me in fornicem esse deductum» $(7,4)$. Si sa che la via che da ignoranza sale a conoscenza non è sempre facile, ma in questo caso l'aspirazione, sebbene scandita dalle voci del verbo scire, non porta ad agnoscere luoghi noti e desiderati, ma si ferma a una comprensione tardiva: inaspettato è il posto d'arrivo che, a giudizio della guida, rappresenta evidentemente il luogo naturale per sfogare l'esuberanza giovanile e che, a giudicare da indizi inequivoci, è comunque riconoscibile agli occhi del mancato cliente.

3 Encolpio reagisce subito all'insidiosa trappola e si dà a fuga precipitosa, ma proprio all'ingresso del lupanare incontra Ascilto a sua volta morto di fatica a dispetto del nome parlante ${ }^{5}$ e reduce in apparenza da un'avventura simile a quella dell'amico ${ }^{6}$. Si rinnova, in effetti, lo scambio tra domanda e risposta, sempre giocato sul problema della conoscenza («quid in loco tam deformi faceret quaesivi»; «si scires [...] quae mihi acciderunt»: $7,5-8,1)$. Ricompare altresì una situazione che conosciamo: anche Ascilto narra che, mentre s'aggirava per la città senza ritrovare la locanda («cum errarem, inquit, per totam civitatem nec invenirem quo loco stabulum reliquissem»: 8, 2), ha incontrato un pater familiae (di solito sinonimo di persona perbene di saldi principi morali), che ha promesso di fargli da guida. Si tratta di promessa fatta humanissime, come suona l'espressione antifrastica che apre uno spazio ironico tra autore e narratore, ma la grande umanità del gentiluomo ha come traguardo il lupanare e come scopo lo stuprum del giovane sorpreso a gironzolare senza meta, comunque in grado di liberarsi dall'insidia mossa alla sua non irreprensibile virtù, perché valentior del molestatore.

4 Bene: la battuta di Encolpio («tarde, immo sero intellexi») segna il passo d'inizio di un motivo che percorre il Satyricon superstite: come si legge nel commento di Gareth Schmeling, "Encolpius' admission of not understanding his situation becames a motif in the Satyrica» ${ }^{7}$. Nella critica petroniana il motivo è stato individuato per tempo: se si vuole restare a un esempio di casa nostra, si può ricordare come nel 1955 Vincenzo Ciaffi (1915-1973) abbia individuato tra le strutture di base della narrazione petroniana la «lunga serie di trappole» in cui incappano i protagonisti, collezionando non meno lunga serie di fallimenti, perché incapaci di comprendere appieno o immediatamente il 
mondo reale che li circonda ${ }^{8}$. A confezionare l'elenco delle incomprensioni e delle comprensioni tardive hanno provveduto e provvedono saggi convincenti che non lasciano dubbi in proposito 9 . Su tale aspetto, pertanto, non è possibile ritornare senza correre il rischio di ripetere il già detto. Pare invece opportuno seguire altra via $\mathrm{e}$ chiederci se alle spalle della 'frase programmatica' pronunciata da Encolpio non sia possibile individuare situazioni paradigmatiche di cui l'autore nascosto'10 operi intenzionali riprese.

5 Proviamo a partire dal mondo greco, dato che la critica non manca di osservare come i fallimenti e le incomprensioni, i ritardi e le delusioni di Encolpio e comprimari facciano rivivere, in forma degradata e parodica, atteggiamenti parziali che connotano molti degli eroi della tradizione teatrale ellenica messi a dura prova da destini di difficile decifrazione. A ben vedere, anzi, nella cultura greca non mancano esempi anteriori alla stessa tradizione teatrale, a cominciare dal mito. Esiste infatti nella mitologia greca una figura che risponde ai requisiti che qui interessano: si tratta di Epimeteo, figlio del titano Giapeto e fratello di Prometeo, entrambi segnati da nome parlante e caratterizzati da epiteti opposti (Vorbedacht e Nachbedacht) ${ }^{11}$, come narra Esiodo: a Giapeto l'Oceanina Climene "generò Atlante, figlio dall'animo forte, / poi partorì il

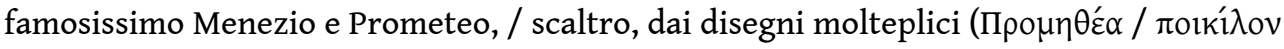

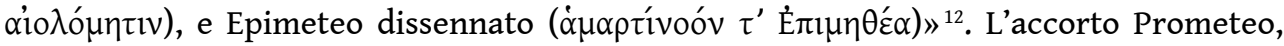
dotato di previdente intelletto, e il maldestro gemello Epimeteo, colui che capisce in

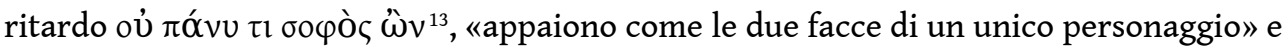
rappresentano opposte qualità ben presenti nella tipologia umana ${ }^{14}$. Insomma, non suona azzardata l'ammissione che Epimeteo, in quanto ó $\psi^{\prime} v o o \zeta^{15} \mathrm{o}$, se si preferisce, slow-thinking ${ }^{16}$, sia l'eponimo di quanti, per ragioni personali o per cause esterne, si trovino nella scomoda posizione di non capire subito la propria situazione e siano costretti a ripetere, in compagnia di un personaggio un tempo assai noto nelle scuole italiane d'ogni ordine e grado, una considerazione poco gratificante: «gran destino è il mio, che le cose a proposito mi vengansempre in mente un momento dopo l'occasione» ${ }^{17}$.

6 Riflessi dell'archetipo di ogni mancata certezza, di ogni pensiero post eventum si possono individuare già nell'epica omerica. Per esempio, nell'Iliade Odisseo, rimasto solo nel cuore della mischia, ha un momento d'incertezza e oscilla tra duplice decisione, fuggire per paura della moltitudine di nemici che lo circonda o lasciarsi catturare, ma sa sfuggire alle opzioni epimeteiche e riconosce infine che è dovere eroico, per chi eccelle in battaglia, restare fermo al proprio posto ${ }^{18}$.

7 All'inizio del XVII canto iliadico, quando sta per scatenarsi la battaglia attorno al cadavere di Patroclo, Menelao si rivolge a Euforbo, il primo a ferire il sodale di Achille, e lo sconsiglia di sfidarlo: «io ti consiglio di fare un passo indietro / e tornare nel folto dei Troiani; non venirmi di fronte / per non conoscere il peggio; lo sciocco capisce solo

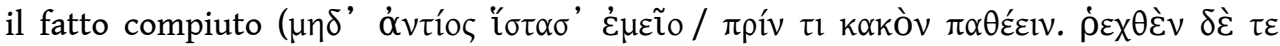
$v \eta ́ \pi ı \varsigma ~ \varepsilon ̌ \gamma v \omega) »^{19}$. All'inizio del XVIII canto iliadico persino Achille, di fronte alla fuga degli Achei verso le navi, comprende in cuor suo quanto ormai è già avvenuto ( $\tau \grave{\alpha}$

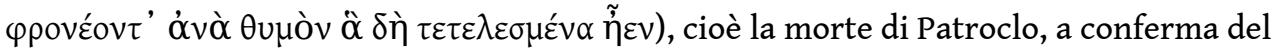
significato d'una profezia materna compresa in ritardo ${ }^{20}$. Il pathos delle tre scene evidenzia in primo piano l'aristia di Menelao, il carattere eroico di Odisseo e l'immenso dolore di Achille, lasciando sullo sfondo l'ombra di processi mentali rallentati. Ma agli eroi dell'Iliade si possono aggiungere le divinità dell'odissea, perché almeno in un caso 
sono due dèi a impersonare condizioni di onniscienza tardiva, per giunta in un contesto che volge al comico. Come è noto, alla corte dei Feaci, dopo l'intermezzo delle gare, Demodoco canta gli amori furtivi di Ares e Afrodite e la vendetta di Efesto, consorte della dea: Odissea, VIII, 266-366 è brano al centro di forte attenzione da parte della critica, che si interroga sulle possibili origini e sulle esegesi più diffuse, dalla possibilità di interpolazione alla mistione tra generi lirici e tradizione epica, dalla presenza di motivi folclorici a suggestioni di ordine religioso ${ }^{21}$. Imprigionati a letto dalle auree catene fabbricate dal marito tradito, sul punto di essere esposti alle risate degli dèi (maschi) d'olimpo, i due amanti «non potevano più muoversi né sollevarsi, / e solo

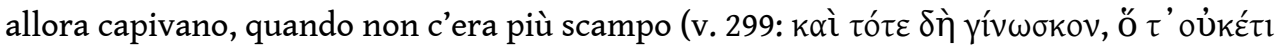

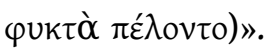

8 Se si dà retta alla Poetica di Aristotele, l'epica appare come matrice fisiologica delle varianti tragiche e comiche del teatro. Nessuna sorpresa, pertanto, se il motivo epimeteico, appena accennato nei poemi, si trasforma in tema ricorrente sulla scena

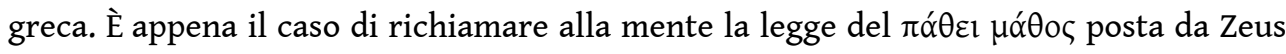
come principio del cammino umano verso saperi pienamente consapevoli, legge evocata dal primo Coro dell'Agamennone di Eschilo ${ }^{22}$. Il dramma della comprensione tardiva è tema drammatico che ha come esempio più complesso la tragedia di Edipo: «In almost everybody's opinion, the Oedipus Rex of Sophocles shows a progression of knowledge in

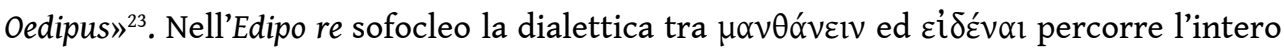
dramma, dal burrascoso scambio tra Tiresia e Edipo, «colui che nulla sa» (v. 397), attraverso le dolorose tappe di conoscenze incomplete o appena intuite (ad es.

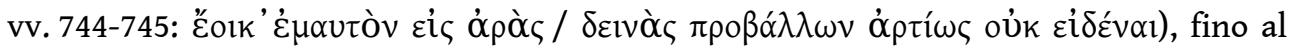
disvelamento operato dalle parole del servo e alla disperata esclamazione del

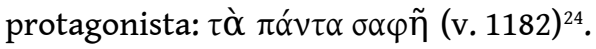

9 A ben vedere, il lento processo di apprendimento, scandito per lo più dalle forme del verbo $\mu \alpha v \theta \alpha ́ v \varepsilon i v$, rappresenta le fasi che preludono alla presa di consapevolezza propria di tutti gli eroi tragici e - si deve aggiungere subito - non assente tra gli eroi comici. Appunto sulla voce verbale appena menzionata prende corpo una formula, $\alpha$ ó $\tau$ $\mu \alpha v \theta \alpha ́ v \omega$, che compare sulla bocca di personaggi pronti ad ammettere la propria tardiva comprensione di quanto accade intorno a loro. La formula è usata da Sofocle nel dramma satiresco Ichneutai (I cercatori di tracce), opera giovanile (?) giunta incompleta e giocata sul furto delle vacche di Apollo, tema dell'Inno omerico a Hermes. Nel corso di un dialogo mal conservato tra i satiri Cercatori di tracce e la ninfa Cillene, nutrice del piccolo Hermes, si salva una battuta non priva d'interesse per il nostro discorso: ó $\rho \tau \imath$

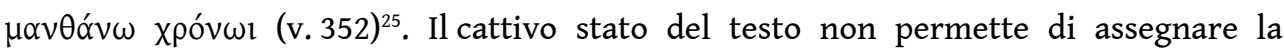
battuta: se fossero di Cillene, le parole sarebbero la tardiva ammissione, da parte della ninfa, delle accuse rivolte dai satiri a Hermes come autore del furto; se invece la battuta si dovesse assegnare al coro (meglio, al corifeo dei Cercatori di tracce), sarebbero i satiri ad ammettere di aver finalmente capito l'enigma di Cillene a proposito del suono derivato da un animale morto, cioè l'invenzione, da parte di Hermes, della lira dal guscio d'una testuggine.

10 A prescindere da ogni ipotesi d'assegnazione, il significato della formula non pare dubbio; con identico valore compare nella prima delle opere euripidee conservate, Alcesti, quarto dramma che ha formato, con Le Cretesi, Alcmeone a Psofide e Telefo, la tetralogia del 438 a.C., sotto l'arcontato di Glaukinos. La vicenda è nota e non necessita di riassunti dettagliati: qui interessa la figura di Admeto, il signore di Fere che ha il 
privilegio di poter vivere della morte altrui ${ }^{26}$, che solo dopo le esequie di Alcesti, morta al posto suo, comprende che il destino della sposa scomparsa è migliore di ciò che lo attende ed esclama: «io, che non dovevo vivere, che ho oltrepassato i limiti del mio

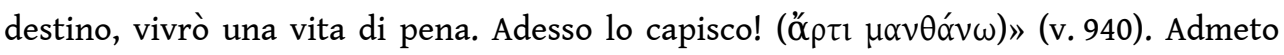
non brilla per conclusioni affrettate: ci vuole infatti quasi tutta la durata del dramma, perché si renda conto di quanto è invece chiaro, nel I episodio, persino a un'ancella

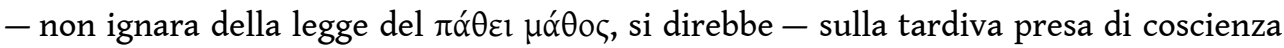

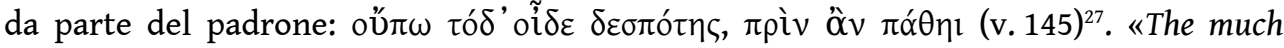
discussed phrase ö $\rho \tau \iota \mu \alpha v \theta \alpha$ ' $v \omega$ (itself a recurrent motif in tragedy) shows that finally Admetos has realised that staying alive at any cost can sometimes be harder than accepting one's fate and dying» ${ }^{28}$.

11 Non importa riaprire in questa sede l'interminabile disputa sulla natura 'prosatiresca'

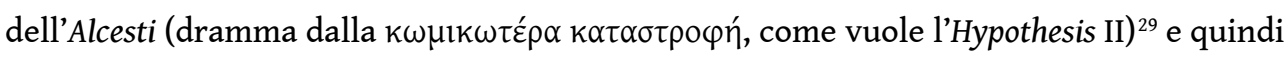
decidere se qui l'uso di ó $\rho \tau \imath \mu \alpha v \theta \alpha ́ v \omega$ sia da intendere in tutto il suo valore di motivo tragico oppure segni di venature parodiche la non impeccabile figura di Admeto; forse a ragione si è scritto che «Alcesti è un'eroina di tragedia, ma ha un marito che viene dalla commedia $»^{30}$. Certo è, però, che Euripide non disconosce il recurrent motif in tragedy,

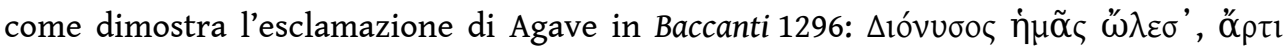
$\mu \alpha v \theta \alpha ́ v \omega$. Tuttavia il motivo può ritornare in indubbi contesti di paratragedia ${ }^{31}$ come elemento di un «conceptual arsenal of tragic ethics (hamartia, arti manthano, pathei mathos, sophrosyne) $\rangle^{32}$ utilizzato anche dai commediografi: valga l'esempio offerto, con opportune variazioni, dal passo delle Vespe di Aristofane, là dove il coro spiega il

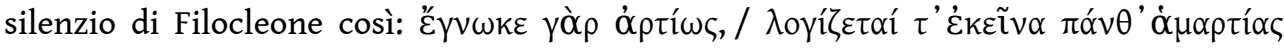
(vv. 744-745) ${ }^{33}$. Non a caso, nelle Nuvole, il futile desiderio d'imparare esibito da Strepsiade si smorza di fronte alla lezione socratica dei nomi maschili e femminili:

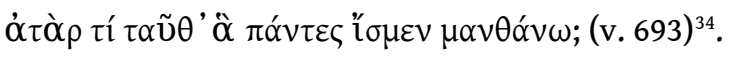

Conferma dell'adozione del motivo da parte degli autori comici viene da Menandro. Per esempio, nel IV atto della Samia ben due personaggi ammettono, a breve distanza scenica, i propri ritardi di comprensione dell'intrigo che li coinvolge. Spetta dapprima a Nicerato apostrofare Moschione come il peggiore di tutti gli uomini e confessare: «In effetti, comincio a rendermi conto, finalmente, di quello che è successo e del

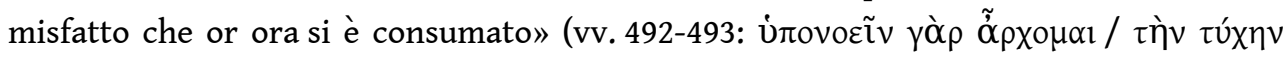

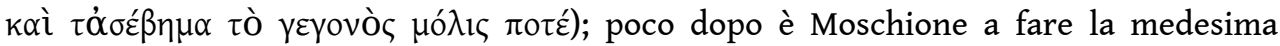
ammissione, rivolgendosi al padre Demea, sordo a ogni ragione: «Solo ora capisco come

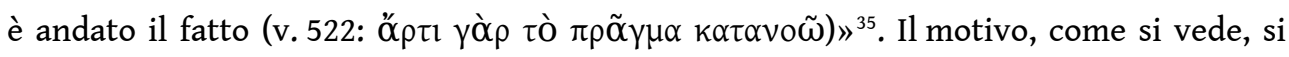
lascia facilmente riconoscere, anche se presentato in vesti lessicali variate; ma a fugare ogni dubbio sulla tenacia, anche in Menandro, della formula vale un frammento

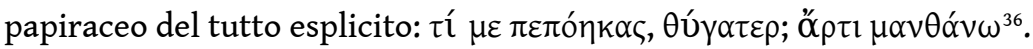

Che il motivo faccia la propria comparsa tra i piani alti della cultura latina non sorprende troppo, in quanto rappresenta un comodo modello per denunciare ritardi e incertezze di comprensione, al fine di manifestare piccole o grandi valutazioni critiche nei confronti di apprendimenti ritardati o di giudizi insoddisfacenti. In proposito soccorre una breve antologia, che può iniziare con un passo di Cicerone: «serius ad te scribo quod sero intellexi quid timendum esset» (Epistole ad Attico, XI, 1, 2) $)^{37}$. Due passi di Seneca non lasciano dubbi sul contesto di forte giudizio morale in cui la formula trova ospitalità. Così nel De traquillitate animi il filosofo invita Sereno (e i lettori d'ogni epoca) a non affaticarsi per traguardi vani e a non desiderare cose impossibili, per non trovarsi 
nell'infelice condizione di capire troppo tardi e a fatica la vanità dei propri desideri (12, 1: «Proximum ab his erit ne aut in supervacuis aut ex supervacuo laboremus, id est ne quae aut non possumus consequi concupiscamus aut adepti vanitatem cupiditatum nostrarum sero post multum sudorem intellegamus, id est ne aut labor irritus sit sine effectu aut effectus labore indignus»). Nel De brevitate vitae la polemica riguarda tutti coloro che ignorano la ricerca filosofica e solo all'ultimo finiscono per prendere atto della vanità dei propri sforzi, accorgendosi di essere stati invano inutilmente affaccendati, anche se hanno trascorso in ozio l'intera esistenza: «Illorum brevissima ac sollicitissima aetas est qui praeteritorum obliviscuntur, praesentia neglegunt, de futuro timent: cum ad extrema venerunt, sero intellegunt miseri tam diu se dum nihil agunt occupatos fuisse» $(16,1)^{38}$.

La ricerca delle analogie espressive che stanno alle spalle della 'frase programmatica' di Encolpio ha dato i suoi frutti: ha individuato una serie di formule che si sono via via precisate, trovando una buona sintesi nelle parole ó $\rho \tau \imath ~ \mu \alpha v \theta \alpha ́ v \omega$ della tradizione teatrale greca; con Seneca le occorrenze approdano all'età neroniana, dunque ai tempi di Petronio, se si accetta l'ipotesi - del tutto prevalente in sede critica - che identifica l'autore del Satyricon con il Petronio arbiter elegantiae alla corte di Nerone ${ }^{39}$. Secondo tale ipotesi, sia Petronio sia Seneca hanno dati biografici largamente confrontabili ed entrambi passano da prossimità alla corte imperiale a forme aperte di dissenso, interpretate in fasi e forme diverse, spesso conflittuali, anche se sempre tributarie di dottrine retoriche ben collaudate ${ }^{40}$. Non solo: nelle opere di entrambi si avverte una indiscussa padronanza della tradizione culturale greco-latina, tradizione accolta con intenti filosofici da Seneca e parodici da Petronio, con l'Apokolokyntosis del primo a far da terreno comune tra i due autori.

Alla luce di quanto detto sin qui, si può tornare là donde si sono prese le mosse, cioè a Petronio 7, 4, ora col sussidio dei precedenti greci e degli esiti in lingua latina della formula ${ }^{41}$. Non sembra possibile decidere per quale via il motivo sia giunto a Petronio e di conseguenza l'ombra di Epimeteo si allunghi fino a coprire i protagonisti del Satyricon; anzi, a ben vedere, è decisione che non mette conto prospettare, perché il lessico latino documentato e gli aspetti indubbiamente teatrali, da teatro comico s'intende, in cui formula e motivo sono calati nel testo petroniano fanno pensare alla coincidenza delle due vie. Che in Petronio si percepisca la volontà di parodiare i poemi omerici (magari intrecciandone episodi, cadenze e funzioni) e, più in generale, si dia spazio a un'epica degradata di eroi di bassa statura etico-sociale capaci di conflitti in sedicesimo, è constatazione acclarata. Ancor più evidenti sono le citazioni e le allusioni virgiliane oppure i riferimenti alla satira e alla tradizione teatrale, tragica, comica e mimica $^{42}$. Si colgono altresì echi della storiografia e della biografia: insomma, a proposito della capacità di ricavare spunti e schegge dai generi della poesia e della prosa, si potrebbe considerare pertinente a Petronio stesso il giudizio espresso nella lezione sulla poesia impartita per strada da Eumolpo: solo una «mens ingenti flumine litterarum inundata» (Petronio 118,3) è in grado di gestire con così tanta abilità un numero così grande di citazioni e di allusioni letterarie altrui. 


\section{BIBLIOGRAFIA}

ALDEN Maureen J., The Resonances of the Song of Ares and Aphrodite, «Mnemosyne», 50, 1997, pp. 513-529.

BALME Maurice (ed.), Menander: The Plays and Fragments (Oxford World's Classics), Oxford, Oxford University Press, 2001.

BARCHIESI Alessandro, Extra legem: consumo di letteratura in Petronio Arbitro, in O. Pecere \& A. Stramaglia (edd.), La letteratura di consumo nel mondo greco-latino, Bari, Levante, 1996, pp. 189-208.

BETTINI Maurizio \& GUIDORIZZI Giulio, Il mito di Edipo. Immagini e racconti dalla Grecia a oggi, Torino, Einaudi, 2004.

BILES Zachary P. \& OLSON S. Douglas (edd.), Aristophanes. Wasps, ed. with Introduction and Commentary, Oxford, Oxford University Press, 2015.

BLAISE Fabienne, JUDET DE LA COMBE Pierre \& ROUSSEAU Philippe (edd.), Le métier du mythe. Lectures d'Hésiode, Lille, Presses universitaires du Septentrion, 1996.

BOMBARDIERI Luca, BRACCINI Tommaso \& ROMANI Silvia (edd.), Il trono variopinto. Figure e forme della Dea dell'Amore, Alessandria, Edizioni dell'Orso, 2014.

BRACCINI Tommaso, Divino scandalo. Gli amori di Ares e Afrodite tra folktales e storie sacre, in L. Bombardieri, T. Braccini \& S. Romani (edd.), Il trono variopinto. Figure e forme della Dea dell'Amore, Alessandria, Edizioni dell'Orso, 2014, pp. 27-46.

BRADLEY Edward M., Admetus and the Triumph of Failure in Euripides' Alcestis, «Ramus», 9, 1980, pp. 112-127.

BRASWELL Bruce Karl, The Song of Ares and Aphrodite: Theme and Relevance to Odyssey 8, «Hermes», 110, 1982, pp. 129-137.

BREITENSTEIN Natalie, Petronius. Satyrica 1-15. Text, Uebersetzung, Kommentar, Berlin / New York, De Gruyter, 2009.

BRILLANTE Carlo, L'Alcesti di Euripide: il personaggio di Admeto e la struttura del dramma, «MD», 54, 2005, pp. 9-46.

BURKERT Walter, Das Lied von Ares and Aphrodite. Zum Verhältnis von Odyssee und Ilias, «RhM», N. F. 103, 1960, pp. 130-144.

CARMIGNANI Marcos, La función de las alusiones a la épica homérica en el Satyricon de Petronio: la “dialogización" y la parodia como criterios de autoridad, «Argos», 32, 2008, pp. 113-133.

CASANOVA Angelo (ed.), Menandro e l'evoluzione della commedia, Firenze, Firenze University Press, 2014.

CERRI Giovanni (ed.), Scrivere e recitare. Modelli di trasmissione del testo poetico nell'antichità e nel medioevo, Roma, Edizioni dell'Ateneo, 1986.

CERRI Giovanni, Omero. Iliade, libro XVIII. Lo scudo di Achille, Roma, Carocci, 2010.

CIAFFI Vincenzo, Struttura del “Satyricon”, Torino, Giappichelli, 1955.

CIAFFI Vincenzo, Petronio. Satyricon. Testo latino a fronte, Torino, Einaudi, 2003. 
COLLIGNON Albert, Étude sur Pétron, la critique littéraire, l'imitation dans le Satiricon, Paris, Hachette, 1892.

CONACHER Desmond J., Euripides. Alcestis, Warminster, Aris \& Phillis, 1988.

CONTE Gian Biagio, L'autore nascosto. Un'interpretazione del “Satyricon”, Bologna, Il Mulino, 1997.

CORAY Marina, Wissen und Erkennen bei Sophokles, Basel / Berlin, Reinhardt, 1993.

COSCI Paola, Per una ricostruzione della scena iniziale del Satyricon, «MD», 1, 1978, pp. 201-207.

COURTNEY Edward (ed.), A Companion to Petronius, Oxford, Oxford University Press, $2003^{2}$.

DI BENEDETTO Vincenzo \& MEDDA Enrico, La tragedia sulla scena. La tragedia greca in quanto spettacolo teatrale, Torino, Einaudi, 1997.

DI SIMONE Marina, I fallimenti di Encolpio, tra esemplarità mitica e modelli letterari: una ricostruzione (Sat. 82, 5; 132, 1), «MD», 30, 1993, pp. 87-108.

DORNSEIFF Franz, Kleine Schriften, vol. I: Antike und alter Orient. Interpretationen, Leipzig, Koeler \& Amelang, 1959.

DÖRRIE Heinrich, Leid und Erfahrung. Die Wort- und Sinn-Verbindung pathein-mathein im griechischen Denken, Wiesbaden, Steiner, 1956.

DOUGHERTY Carol, Prometheus, London / New York, Routledge, 2006.

ENCINAS REGUERO Carmen, El motivo del aprender en Sófocles, «Fortunatae», 23, 2012, pp. 19-30.

FEDELI Paolo, Petronio: il viaggio, il labirinto, «MD», 6, 1981, pp. 91-117.

FERRANDO Serena, Petronius Arbiter Homericae elegantiae, «Maia», 61, 2009, pp. 571-591.

FERREIRA LEAO Delfim, As ironias da fortuna: sátira e moralidade no Satyricon de Petrónio, Coimbra / Lisboa, Colibri, 1998.

FINGLASS Patrick J., Sophocles. Oedipus the King, Cambridge / New York, Cambridge University Press, 2018.

FLOBERT Pierre, Considérations intempestives sur l'auteur et la date du Satyricon sous Hadrien, in J. Herman \& H. Rosén (edd.), Petroniana. Gedenkschrift für Hubert Petersmann, Heidelberg, Winter, 2003, pp. 109-122.

FRAENKEL Eduard, Aeschylus. Agamemnon, vol. II, Oxford, Oxford University Press, 1950.

GUIDORIZZI Giulio \& DEL CORNO Dario (edd.), Aristofane. Le Nuvole, Milano, Fondazione Lorenzo Valla / Mondadori, 1996.

GOLDHILL Simon, The Poet's Voice. Essays on Poetics and Greek Literature, Cambridge, Cambridge University Press, 1991.

GOSTOLI Antonietta, La figura dell'aedo preomerico nella filologia peripatetica ed ellenistica: Demodoco tra mito e storia, in G. Cerri (ed.), Scrivere e recitare. Modelli di trasmissione del testo poetico nell'antichità e nel medioevo, 1986, pp. 103-126.

GUTZWILLER Kathryn, The Tragic Mask: Metatheatricality in Menander, «ClAnt», 19, 2000, pp. 102-137.

HARTIGAN Karelisa V., Ambiguity and Self-Deception. The Apollo and Artemis Plays of Euripides, Frankfurt am Main, Lang, 1991.

HERMAN József \& ROSÉN Hannah (edd.), Petroniana. Gedenkschrift für Hubert Petersmann, Heidelberg, Winter, 2003. 
HESTER David A., The Ignorance of Oedipus, «Prudentia», 25, 1993, pp. 1-23.

HOSE Martin, Euripides. Der Dichter der Leidenschaften, München, Beck, 2008.

JUDET DE LA COMBE Pierre, La dernière ruse: "Pandore" dans la Théogonie, in F. Blaise, P. Judet de La Combe \& P. Rousseau (edd.), Le métier du mythe. Lectures d'Hésiode, Lille, Presses universitaires du Septentrion, 1996.

KISSEL Walter, Petrons Kritik der Rhetorik (Sat. 1-5), «RhM», N. F. 121, 1978, pp. 311-328.

KYRIAKOU Poulheira \& RENGAKOS Antonios (edd.), Wisdom and Folly in Euripides, Berlin / Boston, De Gruyter, 2016.

KNOX Bernard, Oedipus at Thebes. Sophocles' Tragic Hero and His Time, New Haven / London, Yale University Press, 1998.

KOKKINI Dimitra, Euripidean Men Revisited. Four Case Studies, Thesis, University College of London, 2010.

KOTT Jan, Divorare gli dei. Un'interpretazione della tragedia greca, trad. it., Milano, Bruno Mondadori, 2005.

LABATE Mario, Indecifrabilità del reale e prepotenza dell'immaginario nei Satyrica di Petronio, in R. Uglione (ed.), Lector, intende, laetaberis. Il romanzo dei Greci e dei Latini, Alessandria, Edizioni dell'Orso, 2010, pp. 199-217.

LABATE Mario, Tarde, immo iam sero intellexi: The Real as a Puzzle in Petronius' Satyrica, in M. Paschalis \& S. Panayotakis (edd.), The Construction of the Real and the Ideal in the Ancient Novel, Groningen, Barkhuis \& Groningen University Library, 2013, pp. 147-164.

LEFÈVRE Eckard, Die Unfähigkeit, sich zu erkennen: Sophokles' Tragödien, Leiden / Boston / Köln, Brill, 2001.

LESKY Albin, Gesammelte Schriften, Bern / München, Francke, 1966.

MALTESE Enrico V., Sophoclis Ichneutae, Firenze, Gonnelli, 1982.

MARKANTONATOS Andreas, Euripides' Alcestis: Narrative, Myth, and Religion, Berlin / Boston, De Gruyter, 2013.

MARMORALE Enzo V., La questione petroniana, Bari, Laterza, 1948.

MARMORALE Enzo V., Petronio visse tra Commodo ed Elagabalo, «GIF», 2, 1949, pp. 361-367.

MARTIN René, Quelques remarques concernant la date du Satyricon, «REL», 53, 1975, pp. 182-224.

MARTIN René, Qui a (peut-être) écrit le Satyricon?, «REL», 78, 2000, pp. 139-163.

MARTIN René, Petronius Arbiter et le "Satyricon" : quelques pistes de réflexion, «BAGB», 2009, pp. 143-168.

MARTIN René, Le Satyricon est-il un livre à plusieurs mains ?, «REL», 88, 2010, pp. 206-217.

MASTROMARCO Giuseppe, La paratragodia, il libro, la memoria, in E. Medda, M. S. Mirto \& M. P. Pattoni (edd.), Kômôidotragôidia: intersezioni del tragico e del comico nel teatro del V secolo a.C., Pisa, Edizioni della Normale, 2006, pp. 137-191.

MEDDA Enrico, MIRTO Maria Serena \& PATTONI Maria Pia (edd.), Kômôidotragôidia: intersezioni del tragico e del comico nel teatro del V secolo a.C., Pisa, Ed. della Normale, 2006.

MIRALLES MALDONADO José C., La critica della retorica contemporanea nel Satyricon di Petronio, «Maia», 63, 2011, pp. 262-273. 
MORGAN John Robert, Petronius and Greek Literature, in J. Prag \& I. Repath (edd.), Petronius: A Handbook, Chichester / Malden (Mass.) / Oxford, Wiley-Blackwell, 2009, pp. 32-47.

O'BRIEN Michael J. (ed.), Twentieth Century Interpretations of Oedipus Rex: A Collection of Critical Essays, Englewood Cliffs (NJ), Prentice-Hall, 1968.

PALMISCIANO Riccardo, Gli amori di Ares e Afrodite (Od. 8. 266-366). Statuto del discorso e genere poetico, «SemRom», 1, 2012, pp. 187-209.

PANAYOTAKIS Costas, Theatrum Arbitri. Theatrical elements in the Satyrica of Petronius, Leiden / New York / Köln, Brill, 1995.

PANAYOTAKIS Costas, Petronius and the Roman Literary Tradition, in J. Prag \& I. Repath (edd.), Petronius: A Handbook, Chichester / Malden (Mass.) / Oxford, Wiley-Blackwell, 2009, pp. 48-64.

PAOLI Ugo Enrico, L'età del Satyricon, «SIFC», n. s. 14, 1957, pp. 3-46.

PAPADOPOULOU Thalia, Euripidean Frenzy goes to Rome: The Case of Roman Comedy and Novel, in P. Kyriakou \& A. Rengakos (edd.), Wisdom and Folly in Euripides, Berlin / Boston, De Gruyter, 2016, pp. 335-346.

PASCALIS Michael \& PANAYOTAKIS Stelios (edd.), The Construction of the Real and the Ideal in the Ancient Novel, Groningen, Barkhuis \& Groningen University Library, 2013.

PECERE Oronzo \& STRAMAGLIA Antonio (edd.), La letteratura di consumo nel mondo greco-latino, Bari, Levante, 1996.

POIGNAULT Rémy (ed.), Présence du roman grec et latin, Clermont-Ferrand, Centre de recherches André Piganiol - Présence de l'Antiquité, 2011.

POOLE Miriam, Menander's Comic Use of Euripides' Tragedies, «CB», 24, 1978, pp. 111-147.

PRAG Jonathan \& REPATH Ian (edd.), Petronius: A Handbook, Chichester / Malden (Mass.) / Oxford, Wiley-Blackwell, 2009.

PRIULI Stefano, Ascyltus. Note di onomastica petroniana, Bruxelles, Latomus, 1975.

REDONDO Jordi, Alcestis: Pro-Satyric or Simply Romantic Tragedy?, in G. Ugolini, S. Bigliazzi \& F. Lupi

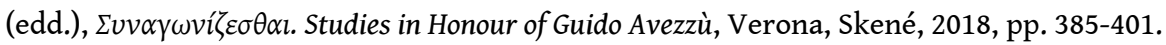

RICCIARDELLI Gabriella (ed.), Esiodo. Teogonia, Milano, Fondazione Lorenzo Valla / Mondadori, 2018.

RIPOLL François, Le Bellum Ciuile de Pétrone : une épopée flavienne ?, «REA»104, 2002, pp. 163-184.

RIPOLL François, Encore sur la datation du Satyricon, in R. Poignault (ed.), Présence du roman grec et latin, Clermont-Ferrand, Centre de recherches André Piganiol - Présence de l'Antiquité, 2011, pp. 439-458.

RUDICH Vasily, Dissidence and Literature Under Nero: The Price of Rhetoricization, Hoboken, Taylor \& Francis, 2013.

SCHMELING Gareth, Quid attinet veritatem per interpretem quaerere? Interpretes and the Satyrica, «Ramus», 23, 1994, pp. 144-168.

SCHMELING Gareth, A Commentary on Satyrica of Petronius, Oxford, Oxford University Press, 2011.

SEGAL Charles, Euripides and the Poetics of Sorrow: Art, Gender, and Commemoration in Alcestis, Hippolytus, and Hecuba, Durham / London, Duke University Press, 1993.

SEGAL Charles, Oedipus Tyrannus. Tragic Heroism and the Limits of Knowledge, New York / Oxford, Oxford University Press, $2001^{2}$. 
SETAIOLI Aldo, L'uso della citazione poetica in Petronio e negli altri romanzieri antichi, «Prometheus», 39, 2013, pp. 188-206.

SICKING Christiaan M. J., Distant Companions: Selected Papers, Leiden / Boston / Köln, Brill, 1998.

SOLIN Heikki, Onomastica petroniana. Il senso nascosto dei nomi nel Satyricon, «Il Nome nel Testo», 19, 2017, pp. 315-328.

SHAW Carl A., Satyric Play. The Evolution of Greek Comedy and Satyr Drama, Oxford, Oxford University Press, 2014.

SOMMERSTEIN Alan, Menander: Samia (The Woman from Samos), Cambridge / New York, Cambridge University Press, 2013.

STAR Christopher, The Empire of the Self: Self-Command and Political Speech in Seneca and Petronius, Baltimore, The Johns Hopkins Press, 2012.

SUSANETTI Davide, Euripide. Alcesti, Venezia, Marsilio, 2001.

TELÒ Mario, Aristophanes and the Cloak of Comedy. Affect, Aesthetics, and the Canon, Chicago / London, The University of Chicago Press, 2016.

UGLIONE Renato (ed.), Lector, intende, laetaberis. Il romanzo dei Greci e dei Latini, Alessandria, Edizioni dell'Orso, 2010.

UGOLINI Gherardo, L'Edipo tragico sofocleo e il problema del conoscere, «Philologus», 131, 1987, pp. 19-31.

UGOLINI Gherardo, BIGLIAZZI Silvia \& LUPI Francesco (edd.), $\Sigma v v \alpha \gamma \omega v i \zeta \varepsilon \sigma \theta \alpha$ l. Studies in Honour of Guido Avezzù, Verona, Skené, 2018.

VEGETTI Mario, Tra Edipo e Euclide. Forme del sapere antico, Milano, Il Saggiatore, 1983.

VERNANT Jean-Pierre, Mito e pensiero presso i Greci. Studi di psicologia storica, trad. it., Torino, Einaudi, 1970.

VERNANT Jean-Pierre, Mito e società nell'antica Grecia, trad. it., Torino, Einaudi, 1981.

VESSEY David W. T., Toughts on the Ancien Novel, or What Ancient? What Novel?, «BICS» 38, 1991-1993, pp. 144-161.

VÖLKER Thomas, Petron und Seneca. Eine Anmerkung zu 'Date and Author of the Satyricon Reconsidered'. Disponibile su <www.academia.edu/15192346/

Petron_und_Seneca._Eine_Anmerkung_zu_Date_and_Author_of_the_Satyricon_reconsidered>.

ZANETTO Giuseppe, La tragedia in Menandro: dalla paratragedia alla citazione, in A. Casanova (ed.), Menandro e l'evoluzione della commedia, Firenze, Firenze University Press, 2014, pp. 83-103.

\section{NOTE}

1. Petronio, Satyricon, 1, 1: «Nunc et rerum tumore et sententiarum vanissimo strepitu hoc tantum proficiunt ut, cum in forum venerint, putent se in alium orbem terrarum delatos» («sbalestrati in un altro mondo» è resa italiana che si deve a Vincenzo Ciaffi). Vedi Cosci (1978, 201-207); Kissel (1978, 311-328); Breitenstein (2009, ad loc.); Miralles Maldonado (2011, 262-273).

2. Petronio, Satyricon, 6, 3: «Sed nec viam diligenter tenebam [quia] nec quo loco stabulum esset sciebam. Itaque quocumque ieram, eodem revertebar». Fa capolino in questo passo il motivo del labirinto, che insieme a quello del viaggio coinvolge e spiega il continuo errare dei protagonisti: vedi ad es. Fedeli (1981, 91-117); Courtney (2003², 227-228). 
3. Petronio, Satyricon, 7, 1: «rogo, inquam, mater, numquid scis ubi ego habitem?».

4. I Glossaria spiegano i tituli come «nomina scortorum et stupri pretium cellis inscripta».

5. "Aeque lassus ac moriens Ascyltos»: il nome deriva dal greco ö $\sigma \kappa v \lambda \tau o \varsigma$, «instancabile» nelle fatiche d'amore. In Petronio, Satyricon, 92,9, là dove si esaltano le dimensioni inguinali del personaggio («inguinum pondus tam grande»), Eumolpo esclama: «o iuvenem laboriosum!» (resa latina dell'antroponimo). Vedi Priuli (1975, passim); Solin (2017, 315-328).

6. Petronio, Satyricon, 7, 4: «cum ecce in ipso aditu occurrit mihi aeque lassus ac moriens Ascyltos; putares ab eadem anicula esse deductum». Per somiglianze e differenze tra le avventure che portano i due protagonisti nel bordello vedi ad es. Ferreira Leão (1998, 76-79).

7. Schmeling $(2011,25)$.

8. Ciaffi $(1955,25)$; testo parzialmente riprodotto come Introduzione in Ciaffi (2003, XI-LIX).

9. Per tutti ci si limita a citare Di Simone (1993, 87-108); Schmeling (1994, 144-168); Labate (2010, 147-164, sull'incapacità degli scholastici di comprendere il mondo esterno e la 'cultura' di Trimalchione e compari); Labate (2013, 199-217).

10. Per la formula si è debitori a Conte (1997).

11. Così F. Dornseiff $(1959,107)$.

12. Esiodo, Teogonia, 509-511; trad. di Ricciardelli $(2018,51)$.

13. Per dirla con Platone, Protagora, 321a.

14. La citazione diretta è di Vernant $(1970,169$, n. 1); in Vernant $(1981,173-191)$ sintesi del mito di Prometeo e le imprese del malaccorto fratello; la considerazione immediatamente successiva deriva da Judet de La Combe (1996, 263-299, in part. 280 sgg.).

15. Pindaro, Pitiche, V, 28.

16. Così Dougherty $(2006,42)$.

17. Ovviamente si tratta del Don Abbondio di manzoniana memoria (I promessi sposi, capitolo I).

18. Omero, Iliade, XI, 401-410.

19. Omero, Iliade, XVII, 30-32 (= Iliade, XX, 196-198, Achille a Enea). L'ultimo emistichio ritorna in

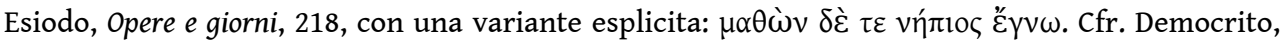
fr. 68 B, 76 D-K: «Per gli sciocchi maestra non è la ragione ma la disgrazia».

20. Omero, Iliade, XVIII, 1-11. Vedi Cerri $(2010,100)$.

21. Il ventaglio delle interpretazioni si ottiene combinando i seguenti contributi: Burkert (1960, 130-144); Braswell (1982, 129-137); Gostoli (1986, 103-126); Alden (1997, 513-529); Palmisciano (2012, 187-209); Braccini (2014, 27-46).

22. Eschilo, Agamennone, 177. Vedi almeno Fraenkel (1950, 106-114); Dörrie (1956, passim).

23. Così Hester $(1993,1)$.

24. Ed. recente: Finglass (2018). Per gli aspetti qui ricordati vedi per es. O'Brien (1968, passim); Vegetti (1983, passim); Ugolini (1987, 19-31); Coray (1993, passim); Di Benedetto \& Medda (1997, 368-389); Knox (1998, passim); Segal (20012); Lefèvre (2001, passim); Bettini \& Guidorizzi (2004, 31-212); Encinas Reguero (2012, 19-30).

25. Sofocle, fr. 314 Radt (inizio di col. XIV di P. Oxy. 1174). Vedi Maltese (1982).

26. Vedi ad es. Brillante (2005, 9-46).

27. Sul ritratto poco lusinghiero di Admeto vedi Lesky (1966, 281-294, Der angeklagte Admet); Bradley (1980, 112-127); Sicking (1998, 48-62); sulla sua «too-late knowledge» vedi almeno Conacher (1988, 191); Hartigan (1991, 32); Segal (1993, 55); D. Susanetti (2001); Hose (2008, 48). Al lessico delle iniziazioni pensa Markantonatos (2013, 150, n. 48).

28. Così Kokkini $(2010,136-137)$.

29. A satyrless satyr play: vedi ad es. Shaw (2014, 78-105); Redondo (2018, 385-401).

30. $\operatorname{Kott}(2005,104)$.

31. Vale a dire la 'messa in commedia' di spunti tragici: vedi ad es. Goldhill (1991, 206-211); Mastromarco (2006, 137-191).

32. Parole di Telò (2016, 82, con discussione del passo citato e di situazioni analoghe). 
33. Vedi Biles \& Olson (2015, ad loc.).

34. Per il doppio senso della battuta, sul genere grammaticale e sulle predilezioni sessuali di Aminias vedi Guidorizzi \& Del Corno $(1996,278)$.

35. Vedi Sommerstein (2013, comm. ad loc.). Sui rapporti tra tradizione tragica e Menandro è utile Gutzwiller (2000, 101-137).

36. Men. Pap. Ghoran II (= P. Sorbonne 72), 101: vedi Balme (2001, 11, Drunkeness - Methe). Per operazioni del genere si rinvia a Poole (1978, 111-147); Zanetto (2014, 83-103).

37. Formula disarticolata in Cicerone, Pro Quinctio, 25: «Pudet dicere; intellego; verum et sero et nequiquam pudet». Cfr. anche l'intervento di Porcio Latrone in Seneca Padre, Controversiae, VII, 7, 7: «vereor, ne tam sero caveamus quam imperator noster, qui non ante intellexit proditionem quam proditus est».

38. Se fosse sicura la datazione ai tempi di Caligola o di Claudio dell'opera superstite di Curzio Rufo, potrebbe tornare di qualche utilità un passo del decimo libro delle Historiae Alexandri Magni: «Meleager, sero intellecta fraude Perdiccae, tum quidem, quia ipsius corpori vis non adferebatur, in agmine quietus stetit» (X, 9: sull'inizio della guerra tra i successori di Alessandro Magno).

39. Per datazione di autore e testo non mancano altre proposte, come mostra l'elenco di Collignon (1892, 343-345), che pensa ai tempi di Domiziano o di Traiano. Nel Novecento Marmorale (1948, 24; 1949, 361-367) data il Satyricon tra Commodo ed Elagabalo; Paoli (1957, 3-46) lo data agli inizi del II sec. d.C. All'età di Domiziano pensa Martin (1975, 182-224); all'età di Vespasiano Vessey (1991-1993, 144-161); all'età di Traiano Martin (2000, 182-224; 2009, 143-168); all'età di Adriano Flobert (2003, 109-122), all'età flavia Ripoll (2002, 163-184; 2011, 439-458). Per Martin $(2010,206-217)$ il testo sarebbe opera di più mani distanti nel tempo. Su tale ventaglio di proposte prevale l'opinione di Courtney $\left(2003^{2}, 11\right)$ : «nessuno degli argomenti a favore dell'identificazione col personaggio neroniano costituisce una prova, ma a giudizio della maggior parte della critica moderna l'insieme va accettato come un'ipotesi ragionevole».

40. Vedi ad es. Star (2012, passim); Rudich (2013, passim). I dati bibliografici sui rapporti tra Seneca e Petronio sono raccolti in due dense pagine di Völker (academia.edu, 2016).

41. Non è forse inutile ricordare che l'esito latino compare nell'Institutio oratoria di Quintiliano all'interno della discussione sulla nozione di urbanitas proposta da Domizio Marso: «Neque enim ei de risu sed de urbanitate est opus institutum, quam propriam esse nostrae civitatis et sero sic intellegi coeptam, postquam urbis appellatione, etiam si nomen proprium non adiceretur, Romam tamen accipi sit receptum» (VI, 3, 103).

42. Vedi Panayotakis (1995); Barchiesi (1996, 189-208); Carmignani (2008, 113-133); Ferrando (2009, 571-591); Morgan (2009, 32-47); Panayotakis (2009, 48-64); Setaioli (2013, 188-206). Per alcuni spunti circa le riprese in autori latini e in Petronio di motivi derivati dalle Baccanti di Euripide vedi Papadopoulou (2016, 335-346).

\section{RIASSUNTI}

In Satyricon, 7, 4 sgg. Encolpio e Ascilto si accorgono in ritardo di essere finiti in un lupanare. Secondo la critica moderna la scenetta prefigura un motivo che percorre il testo superstite di Petronio: l'incapacità, da parte degli scholastici, di comprendere subito il mondo reale che li circonda. Nell'articolo si ricercano i precedenti della battuta di Encolpio (tarde, immo sero intellexi). Eponimo mitico di chi tarda a capire è Epimeteo, il maldestro gemello di Prometeo; 
situazioni e lessico trovano nell'epica omerica e nella tradizione teatrale esempi di eroi segnati da comprensioni tardive o insufficienti. La formula ö $\rho \tau \imath \mu \alpha v \theta \alpha ́ v \omega$ ed espressioni greche equivalenti passano al lessico latino attraverso varianti di sero intellegere. La ripresa presente nel passo di Petronio conferma la cultura dell'autore e la sua abilità nel fare tesoro di citazioni e allusioni letterarie.

Dans Satyricon, 7, 4 ss., Encolpe et Ascylte comprennent tardivement qu'ils se trouvent dans un lupanar. Ce qui se passe sur la scène préfigure un motif récurrent dans le texte de Pétrone, à savoir l'inaptitude des scholastici à saisir rapidement le monde réel qui les entoure. Dans cette contribution, nous nous proposons de rechercher les antécédents des mots d'Encolpe (tarde, immo sero intellexi). De son côté, Épiméthée, le jumeau maladroit de Prométhée, est l'éponyme mythique de ceux qui comprennent en retard ; les situations et le lexique trouvent dans l'épique homérique et plus encore dans la tradition théâtrale de nombreux exemples de héros qui sont marquées par une compréhension tardive ou insuffisante. La tournure ö $\rho \tau \iota \mu \alpha v \theta \alpha ́ v \omega$ et d'autres expressions grecques équivalentes sont reçues dans le lexique latin et sont traduites par des variantes de sero intellegere. La reprise dans le passage de Pétrone témoigne une fois de plus de la culture de l'auteur et de sa capacité à tirer profit des citations et des allusions littéraires.

In Satyricon, 7, 4 ff., Encolpius and Ascyltos realize too late that they have come to a brothel. Modern scholars have argued that this scene prefigures a motif running through all of the surviving text by Petronius: the schoolmen's failure to immediately understand the real world around them. This paper aims to discuss the precedents of Encolpius' words tarde, immo sero intellexi. Epimetheus, Prometheus' awkward twin brother, is the mythical name for a person who is slow-witted; situations and lexical examples found in the Homeric epic and in the theatrical tradition involve heroes in events characterized by belated or unsatisfactory understanding. Both the formula $\alpha$ prı $\mu \alpha v \theta \alpha v \omega$ and similar Greek sentences have passed into the Latin lexicon, and are realised as variants of sero intellegere. The reference in Petronius's passage confirms the author's culture and his ability to draw upon quotations and literary allusions.

\section{INDICE}

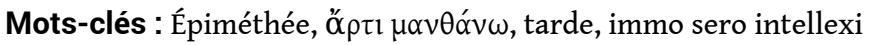

Parole chiave : Epimeteo, ó $\rho \tau \iota ~ \mu \alpha v \theta \alpha ́ v \omega$, tarde, immo sero intellexi

Keywords : Epimetheus, ő $\rho \tau \imath \mu \alpha v \theta \alpha ́ v \omega$, tarde, immo sero intellexi

\section{AUTORE}

\section{GIAN FRANCO GIANOTTI}

Accademia delle Scienze di Torino

gianfrancogianotti3@gmail.com 